\title{
Role of medicinal plants against Alzheimer's disease
}

\begin{abstract}
Alzheimer's is an irreversible and dynamic neurodegenerative ailment which associated with the age and characterized by serious loss of memory, personality changes, uncommon behaviour and decrease in intellectual capacity. Till now no countermeasure available for Alzheimer's, however symptomatic treatment may enhance memory and other issues related to this disease. Products from natural resources such as medicinal plants have been utilized for the treatment of various memory disorders like amnesia, dementia, Alzheimer's, Parkinson since long time. Various studies depicted the utilization of therapeutic plants for treatment of Alzheimer's. In spite of that exact mode of their action is yet indistinct. Phytochemical investigations of various plant parts have demonstrated presence of numerous bioactive compounds, such as polyphenols, tannins, flavonoids, triterpenes, alkaloids and sterols. These compounds demonstrate an extensive variety of pharmacological activities which includes anticholinesterase, antiinflammatory, antiamyloidogenic, hypolipidemic, and antioxidant effects. This review provides the description of different medicinal plants against Alzheimer's disease.
\end{abstract}

Keywords: medicinal plants, Alzheimer's disease, phytocompounds
Volume II Issue 4 - 2018

\author{
Arpita Roy \\ Delhi Technological University, India
}

Correspondence: Arpita Roy, Delhi Technological University, New Delhi-II0042, India, Email arbt20|4@gmail.com

Received: April 20, 2018 | Published: July 12, 2018

\section{Introduction}

Neurodegenerative ailments are heterogeneous group of genetic disorders that are indicated by loss of neuronal structure, function and generally lead to neuronal loss. These ailments may result directly from particular neuronal population degeneration or indirectly from changes in glial support cells. These kinds of ailments are characterized by an abnormal proteins accumulation or other biological materials accumulation within or outside the neurons. These aggregations take different structures and result in neurofabrillary tangles in Alzheimer's disease, Lewy bodies in Parkinson's disease, glycogen and polyglucosan bodies in Lafora disease. Alzheimer's disease is thought to be in responsible of $60 \%$ of all mental disorder in moderately aged or older adults ${ }^{1}$ and influencing in excess of 5 million Americans, a number assessed to increment to 7.7 million by 2030 .

Alzheimer's disease is caused due to aggregation of mis-folded proteins which accumulate fibrillary amyloid deposits in selective regions of central nervous system. Alzheimer's disease results in memory loss, personality changes, unusual behaviour, and loss of the ability to thinking. Early disease shows short term memory loss, fail to remember names and addresses, failure to learn new information, mood swings, as the condition develops, changes become more prominent and individuals even fail to remember way to home. Frustration, irritability and hostility are usual emotional features showed by AD patients. Seventy percent of Alzheimer's disease is caused due to genetic factor and twenty one percent is environmental factor. Most cases of Alzheimer's disease are late-onset which develops after a age of $60 .{ }^{2}$ At present, most accepted Alzheimer's disease treatment strategy is inhibitors of cholinesterase which can inactivate acetylcholinesterase (AChE) enzyme in order to enhance the acetylcholine levels in brain. Inhibitors of acetylcholinesterase include rivastigmine, donepezil, tacrine, galantamine and memantine. However, there is no drug for Alzheimer's disease, besides only calm the disease symptoms. ${ }^{3}$ Herbal medicine offers an alternative option to modify symptoms and progress of $\mathrm{AD}$.
Products that are derived from plants are carefully standardized, and their safety and efficacy for a particular application have been tested. ${ }^{4}$ Herbal medicine is indigenous to India and in Ayurveda several preparations of medicine have been developed for various disease treatments. Various scientific investigations have shown the importance of medicinal plants in the enhancement of nervous system function. ${ }^{5}$ Phytochemical analysis reveals the presence of various bioactive compounds, such as tannins, lignans, polyphenols, flavonoids, sterols, triterpenes and alkaloids in medicinal plants which shows the potential against various problems, including antiamyloidogenic, anti-inflammatory, anti-cholinesterase, hypolipidemic, and antioxidant effects. ${ }^{6}$ This review provides the description of medicinal plants that possesses promising role in Alzheimer.

\section{Medicinal plants against Alzheimer's disease}

Medicinal plants contain various phytocompounds which are extractable and utilized as raw material for different scientific survey. Various secondary metabolites from plants are commercially essential and utilized in pharmaceutical industries. Recently, medicinal plants have gained wide acceptance because of thier fewer side effects compared to the synthetic medicines and necessity to meet the requirement of medicine for increasing human population. However, steady supply of source material often becomes difficult due to various factors like diverse geographical distribution, environmental changes, cultural practices, labour cost, selection of superior plant stock and over exploitation by pharmaceutical industries. ${ }^{7}$ Several investigations reported that medicinal plants are utilized in the Alzheimer's disease treatment which includes Centella asiatica, Ginkgo biloba, Withania somnifera, Bacopa monnieri, Salvia officinalis, Melissa officinalis, Tinospora cordifolia, Glycyrrhiza glabra etc.

\section{Withania somnifera (Ashwagandha)}

Withania somnifera is largely utilized in Ayurveda as a nerve tonic which supports the body to adapt stress. W. somnifera belongs to Solanaceae family, root of this plant is extensively utilized. It possesses free radical scavenging activity, antioxidant activity, 
and enhances immune system. ${ }^{8}$ W. somnifera possesses a calming effect whereas other adaptogens tends to stimulate and thus shows positive effects in people with Alzheimer's disease. ${ }^{9}$ A recent study of $W$. somnifera showed decreased level of stress and inability to concentrate and reversed forgetfulness in a dose-dependent manner in and there is no adverse effects were found [10]. W. somnifera contains various phytocompounds which include which includes withanolides A to $\mathrm{Y}$, withasomidienone, withasomniferin A, dehydro withanolide $\mathrm{R}$, withaferin A, withasomniferols A to C, and withanone, phytosterols sitoindosides VII to X, beta-sitosterol, alkaloids, amino acids and high amounts of iron. ${ }^{11}$ Withanamides showed free radicals scavenge activity that generated during the Alzheimer's disease initiation and progression. It blocked the death of neuronal cell triggered by amyloid plaques. ${ }^{12}$ Molecular modelling investigations showed that withanamides $A$ and $C$ particularly bind to $\beta$-amyloid (A $\beta$ 25-35) active motif and prevents the formation of fibril. ${ }^{13}$ Aqueous extracts of $W$. somnifera showed the enhanced cholinergic activity by increasing the acetylcholine content and cholineacetyl transferase activity in rats, this shows the cognition-enhancing and memory-improving effects. ${ }^{14}$ In addition to pre- and post-synapses reconstruction in the neurons, methanol extracts of $W$. somnifera reversed the amyloid peptide-induced memory deficit in mice. ${ }^{15}$ These in vivo effects of $W$. somnifera were continuing even after drug termination.

\section{Bacopa monnieri (Brahmi)}

Bacopa monnieri belongs to Scrophulariaceae family and found in marshy and damp areas. It is commonly utilized in Ayurvedic medicine and act as a nerve tonic, cardiotonic, diuretic and as therapeutic agent against asthma, insomnia, epilepsy, and rheumatism. This plant contains various phytocompounds which includes bacosides $\mathrm{A}$ and $\mathrm{B}$, bacopasides III to $\mathrm{V}$, and bacosaponins $\mathrm{A}, \mathrm{B}$, and $\mathrm{C}$, jujubogenin bisdesmosides bacopasaponins D, E, and F, betulic acid, sterols, alkaloids, polyphenols, and sulfhydryl compounds that suggested its antioxidant activity. ${ }^{16}$ Traditionally, B. monnieri was utilized for memory and cognitive function enhancement. Extracts of $B$. monnieri has been extensively investigated for its neuropharmacological effects and nootropic actions. ${ }^{17}$ In hippocampus, B. monnieri increase the protein kinase activity which contributes to its memory enhancing properties..$^{18}$ B. monnieri also inhibited cholinergic degeneration and shows enhanced cognition effect in Alzheimer model of rat. ${ }^{19}$ It was reported that a standardized $B$. monnieri extract reversed the cognitive deficits induced by intra-cerebroventricularly administered colchicines and ibotenic acid into the nucleus basalis magnocellularis. ${ }^{20}$ In the same investigation, B. monnieri also reversed the acetylcholine depletion, choline acetyltransferase activity reduction, and reduce the muscarinic cholinergic receptor binding in frontal cortex of hippocampus..$^{20} \mathrm{~B}$. monnieri extracts protected the neurons from $\beta$ amyloid induced cell death by repressing cellular acetylcholinesterase activity. B. monnieri extract treated neurons showed lower reactive oxygen species level which suggested that B. monnieri restrained intracellular oxidative stress. ${ }^{21}$

\section{Centella asiatica (Gotu Kola)}

Centella asiatica belongs to Apiaceae family and found throughout India also in Sri Lanka and Bangladesh. ${ }^{22}$ It contains various bioactive compounds which include triterpenes, asiatic acid, asiaticoside, adecassoside, sapogenins, glycosides, madecassic acid, vellarin, and centelloside. ${ }^{23}$ Asiatic acid and asiaticoside showed reduce hydrogen peroxide-induced cell death, decline concentration of free radicals, and $\beta$-amyloid cell death inhibition in vitro which suggested possible role in Alzheimer's disease treatment and $\beta$-amyloid toxicity prevention. ${ }^{24-26}$ Extracts of Centella asiatica reversed the $\beta$-amyloid pathology in mice brains and modulated oxidative stress response components. ${ }^{27}$ It is an important plants for nerve and brain cells and considers being capable of enhancing intellect, memory and longevity. ${ }^{17}$

\section{Ginkgo biloba}

Ginkgo biloba belongs to Ginkgoaceae family and indigenous to China. Extract of G. biloba used to treat inadequate blood circulation complications, consciousness loss, depression and headaches. ${ }^{28}$ This extract was accounted to contain around $24 \%$ of flavonoids and $6 \%$ of terpene lactones. There is strong evidence that standardized ginkgo extract demonstrates several molecular and cellular neuroprotective mechanisms, which include apoptosis attenuation, membrane lipid peroxidation inhibition, anti-inflammatory effects and $\beta$-amyloid aggregate inhibition. There are considerable clinical examinations in regards to its potential role in cognitive disorders. ${ }^{28}$ Chronic treatment on learning and memory in mice demonstrated that G. biloba enhanced acquisition, storage, and retrieval of two-response sequence for food reward. ${ }^{29}$ G. biloba influences intellectual capacity in Alzheimer's disease animal model without altering histopathological consequences of $\beta$ amyloid precursor protein overexpression. ${ }^{30} G$. biloba extract significantly repress acetylcholinesterase activity in the brain and $\mathrm{AChE}$ activity inhibition corresponded with improvement scopolamine-induced deficits in passive avoidance. Decline acetylcholinesterase activity indicates enhance basal level of acetylcholine. ${ }^{31}$

\section{Curcuma longa (Turmeric)}

Curcuma longa belongs to Zingiberaceae family and possesses anti-inflammatory activity which is also associated with reduced Alzheimer's risk. ${ }^{32}$ Curcumin reduces deposition of plaque in the brain. It decreases oxidative stress and amyloid pathology. ${ }^{5}$ Epidemiologic studies showed that in Southeast Asian countries where turmeric is frequently utilized in diet there are 4.4-fold lower cases of Alzheimer. ${ }^{33}$ A study reported that low doses of Curcumin reduced A $\beta$ level up to $40 \%$ in mice with Alzheimer's disease as compared to control drug. At lower dose curcumin caused $43 \%$ decrease in plaque burden that these $A \beta$ have on the brain of mice with Alzheimer's disease. ${ }^{5}$ Another study indicates that anti-inflammatory property of turmeric is correlated with a decrease Alzheimer risk. ${ }^{34}$

\section{Glycyrrhiza glabra}

Glycyrrhiza glabra belongs to Fabaceae family and contains various bioactive compounds which include linalool oxide, geraniol, benzoic acid, terpinen, tetramethyl pyrazine, propionic acid, ethyl linolenate, butanediol, feuferaldehyde, methyl ethyl ketone, furfuryl formate, trimethylpyrazine, glycyrrhizin, tannin, and glycyrrhizic acid. ${ }^{35}$ Memory enhancing activity of this plant was reported in scopolamine induced dementia. ${ }^{36}$ Dhingra et al. ${ }^{37}$ reported that in mice Glycyrrhiza glabra enhances memory and three dose levels (75, $150,300 \mathrm{mg} / \mathrm{kg}$ ) of plant extracts were administered to mice in seven successive days and dose at $150 \mathrm{mg} / \mathrm{kg}$ was found effective in memory enhancement. This could be helpful in the treatment of AD. 


\section{Lepidium meyenii}

It belongs to Brassicaceae family and known for its improving learning abilities and memory function. ${ }^{38}$ It exhibited memory enhancing activity in Alzheimer's patients. It enhances memory by increasing the acetylcholine level. ${ }^{39}$ It enhances memory impairment which was induced by ovariectomy, due in part to its acetylcholinesterase inhibitory and antioxidant effects. ${ }^{40}$

\section{Magnolia officinalis}

It belongs to the family Magnoliaceae and improves scopolamine induced memory deficits. It inhibits acetyl cholinesterase activity. ${ }^{41}$ Magnolol and honokiol derived from Magnolia officinalis, have the ability to enhance choline acetyltransferase effects. Also inhibits the acetylcholine cleavage and have shown to release acetylcholine from hippocampus. ${ }^{42}$ Honokiol exhibits anti-inflammatory activity by reactive oxygen species synthesis inhibition. ${ }^{43}$ Anti-inflammatory and antioxidant activity of Magnolia officinalis plays vital role in the Alzheimer treatment. ${ }^{44}$

\section{Tinospora cordifolia (Giloy)}

Tinospora cordifolia belongs to Menispermaceae family and possesses memory enhancing properties in normal and memory lack animals. It enhance cognitive by immune-stimulation and increasing acetylcholine synthesis, this choline supplementation increase cognitive function. In Ayurveda Tinospora cordifolia is known as learning and memory enhancer. Aqueous extract of Tinospora cordifolia roots showed verbal learning and logical memory enhancement. ${ }^{45}$

\section{Convolvulus pluricaulis (Shankhpushpi)}

Convolvulus pluricaulis belongs to Convolvulaceae family and used as a memory enhancer. A study has shown that aqueous extract and ethyl acetate of Convolvulus pluricaulis increases memory functions and learning abilities. ${ }^{46}$ This plant has been reported to calm nerves by regulating stress hormones synthesis like cortisol and adrenaline in the body ${ }^{47}$ Ethanolic extract of this plant also significantly improved learning abilities and memory retention in rats. ${ }^{48}$ Administration of Convolvulus pluricaulis increased acetylcholinesterase activity in hippocampal CA1 and CA3 regions associated with the memory function and learning abilities. ${ }^{49}$

\section{Conclusion}

Interests in the utilization of different herbal products increase day by day. As several studies show that utilization of synthetic drugs have side effects, so there is a need of alternative source of drugs which have low or negligible side effects. Medicinal plants contain wide range of bioactive compounds which is an alternative of synthetic drug for Alzheimer's disease treatment. Medicinal plants can boost life quality of patients with $\mathrm{AD}$ and memory deficits. This review provides the details about the role of medicinal plants against the Alzheimer's disease. However, mechanisms of action are still not clear. Future clinical trials involving larger sample sizes are require investigating role of different medicinal plants and the underlying mechanisms.

\section{Acknowledgements}

None.

\section{Conflict of interest}

Author declares there is no conflict of interest towards this manuscript.

\section{References}

1. Francis PT, Plamer AM, Anape M, et al. The cholinergic hypothesis of Alzheimer's dieases:-A review of progress. J Neurol Neurosurg Psychiatry. 1999;66(2):137-147.

2. Herring A, Ambrée O, Tomm M, et al. Environmental enrichment enhances cellular plasticity in transgenic mice with Alzheimer-like pathology. Exp Neurol. 2009;216(1):184-192.

3. Orhan I, Aslan M. Appraisal of scopolamine-induced antiamnesic effect in mice and in vitro antiacetylcholinesterase and antioxidant activities of some traditionally used Lamiaceae plants. $J$ Ethnopharmacol. 2009;122(2)327-332.

4. Kennedy DO, Wightman EL. Herbal extracts and phytochemicals: plant secondary metabolites and the enhancement of human brain function. $A d v$ Nutr. 2011;2(1):32-50.

5. Mishra S, Palanivelu K. The effect of Curcumin (turmeric) on Alzheimer's disease: An overview. Ann Indian Acad Neuro. 2008;11(1):13-19.

6. Howes MJ, Perry NS, Houghton PJ. Plants with traditional uses and activities, relevant to the management of Alzheimer's disease and other cognitive disorders. Phytother Res. 2003;17(1):1-18.

7. Joy PP, Thomas J, Mathew S. Medicinal Plants. Tropical Horticulture. 2001; 449-632 p.

8. Russo A, Izzo AA, Cardile V, et al. Indian medicinal plants as antiradicals and DNA cleavage protectors. Phytomedicine. 2001;8(2):125-132.

9. Monograph. Withania somnifera. Alternative Medicine Review. 2004;9(2):211-214

10. Auddy B, Hazra J, Mitra A, et al. A standardized Withania somnifera extract signifi cantly reduces stress-related parameters in chronically stressed humans: a double-blind randomized, placebocontrolled study. JANA. 2008;11(1):50-56.

11. Matsuda H, Murakami T, Kishi A, et al. Structures of withanosides I, II, III, IV, V, VI, and VII, new withanolide glycosides, from the roots of Indian Withania somnifera DUNAL and inhibitory activity for tachyphylaxis to clonidine in isolated guinea-pig ileum. Bioorg Med Chem. 2001;9(6):1499-1507.

12. Jayaprakasam B, Padmanabhan K, Nair MG. Withanamides in Withania somnifera fruit protects $\mathrm{PC}-12$ cells from beta-amyloid responsible for Alzheimer's disease. Phytother Res. 2010;24(6):859-863.

13. Kumar S, Harris RJ, Seal CJ, et al. An aqueous extract of Withania somnifera root inhibits amyloid beta fibril formation in vitro. Phytother Res. 26(1):113-117.

14. Schliebs R, Liebmann A, Bhattacharya SK, et al. Systemic administration of defi ned extracts from Withania somnifera (Indian Ginseng) and Shilajit differentially affects cholinergic but not glutamatergic and GABAergic markers in rat brain. Neurochem Int. 1997;30(2):181-190.

15. Kuboyama T, Tohda C, Komatsu K. Neuritic regeneration and synaptic reconstruction induced by withanolide A. Br J Pharmacol. 2005;144(7):961-971.

16. Monograph. Bacopa monniera. Alternative Medicine Review. 2004;9(1):79-85.

17. Shinomol GK, Muralidhara, Bharat MM. Exploring the role of 'Brahmi' (Bacopa monnieri and Centella asiatica) in brain function and therapy. Recent Pat Endocr Metab Immune Drug Discov. 2011;5(1):33-49. 
18. Singh HK, Dhawan BN. Effect of Bacopa monniera Linn. (brahmi) extract on avoidance responses in rat. J Ethnopharmacol. 1982;5(2):205214.

19. Uabundit N, Wattanathorn J, Mucimapura S, et al. Cognitive enhancemen and neuroprotective effects of Bacopa monnieri in Alzheimer's disease model. J Ethnopharmacol. 2010;127(1):26-31.

20. Bhattacharya SK, Bhattacharya A, Kumar A, et al. Antioxidant activity of Bacopa monniera in rat frontal cortex, striatum and hippocampus. Phytother Res. 2000;14(3):174-179.

21. Limpeanchob N, Jaipan S, Rattana karuna S, et al. Neuroprotective effect of Bacopa monnieri on beta-amyloid-induced cell death in primary cortical culture. J Ethnopharmacol. 2008;120(1):112-127.

22. Roy A, Bharadvaja N. Effect of Different Culture Medias on Shoo Multiplication and Stigmasterol Content in Accessions Of Centella Asiatica. International Journal of Ayurvedic \& Herbal Medicine. 2017;7:2643-2650.

23. Roy A, Bharadvaja N. Centella Asiatica: A Pharmaceutically Important Medicinal Plant. Curr Trends Biomedical Eng \& Biosci. 2017;5(3): 555662.

24. Dhanasekaran M, Holcomb LA, Hitt AR, et al. Centella asiatica extract selectively decreases amyloid beta levels in hippocampus of Alzheimer's disease animal model. Phytother Res. 2009;23(1):14-19.

25. Roy A, Kundu K, Saxena G, Bharadvaja N. Estimation of Asiaticoside by Using RP-HPLC and FAME Analysis of Medicinally Important Plant Centella asiatica. J Plant Biochem Physiol. 2017;5:198.

26. Roy A, Kundu K, Saxena G. Effect of different media and growth hormones on shoot multiplication of in vitro grown Centella asiatica accessions. Adv Tech Biol Med. 2016;4:172.

27. Cervenka F, Jahodar L. Plant metabolites as nootropics and cognitives Ceska Slov Farm. 2006;55(5):219-229.

28. Luo Y. Alzheimer's disease, the nematode Caenorhabditis elegans, and Ginkgo biloba leaf extract. Life Sci. 2006;78(18):2066-2072.

29. Winter E. Effects of an extract of Ginkgo biloba on learning and memory in mice. Pharmacol Biochem Behav. 1991;38(1):109-114.

30. Stackman RW, Eckenstein F, Frei B, et al. Prevention of age-related spatial memory deficits in a transgenic mouse model of Alzheimer's disease by chronic Ginkgo biloba treatment. Exp Neurol. 2003;184(1):510-520.

31. Das A, Shanker G, Nath C, et al. A comparative study in rodents of standardized extracts of Bacopa monniera and Ginkgo biloba: anticholinesterase and cognitive enhancing activities. Pharmacol Biochem Behav. 2002;73(4):893-900.

32. Aggarwal B, Harikumar K. Potential therapeutic effects of Curcumin, the anti-inflammatory agent, against neurodegenerative, cardiovascular, pulmonary, metabolic, autoimmune and neoplastic diseases. Inter $J$ Biochem Cell Biol. 2009;41(1):40-59.

33. Ganguli M, Chandra V, Kamboh MI, et al. Apolipoprotein E polymorphism and Alzheimer disease: The Indo-US Cross-National Dementia Study. Arch Neurol. 2000;57(6):824-830.
34. Breitner JC, Welsh KA, Helms MJ, et al. Delayed onset of Alzheimer's disease with nonsteroidal anti-inflammatory and histamine $\mathrm{H} 2$ blocking drugs. Neurobiol Aging. 1995;16(4):523-530.

35. Rekha S, Parvathi A. Evaluation of phytochemical constituents of the roots of Licorice, Indian ginseng, Indian madder and Indian Sarasaparilla. Biochemistry. 2012;3(2):357-361.

36. Ambawade S, Kasture V, Kasture S. Anxiolytic activity of Glycyrrhiza glabra linn. J Nat Remed. 2009;1(2):130-134.

37. Dhingra D, Parle M, Kulkarni S. Memory enhancing activity of Glycyrrhiza glabra in mice. J Ethnopharmacol. 2004;91(2-3):361-365.

38. Julio R, Dang H, Gong M, et al. Aqueous and hydroalcoholic extracts of black maca (Lepidium meyenii) improve scopolamine-induced memory impairment in mice. Food Chem Toxico. 2007;45(10):1882-1890.

39. Wang R, Yan H, Tang X. Progress in studies of huperzine A, a natural cholinesterase inhibitor from Chinese herbal medicine. Acta Pharmacol Sin. 2006;27(1):1-26.

40. Rubio J, Qiong W, Liu X, et al F. Aqueous extract of black maca (Lepidium meyenii ) on memory impairment induced by ovariectomy in mice. Evid Based Complement Alternat Med. 2011;2:1-5.

41. Jae L, Kyung L, Beom J, et al. Inhibitory effect of ethanol extract of Magnolia officinalis and 4-O-methylhonokiol on memory impairment and neuronal toxicity induced by beta-amyloid. Pharmacol Biochem Behav. 2009;95(1):31-40

42. Hou YC, Chao PD, Chen YT. Honokiol and magnolol increased hippocampal acetylcholine release in freely moving rats. Am J Chin Med. 2000;28(3-4):379-384.

43. Dikalov S, Losik T, Arbiser J. Honokiol is a potent scavenger of superoxide and peroxyl radicals. Biochem Pharmacol. 2008;76(5):589-596.

44. Liou KT, Shen YC, Chen CF, et al. The anti-inflammatory effect of honokiol on neutriprils: mechanism in the inhibition of reactive oxygen species production. Eur J Pharmacol. 2003;47(1-3):19-27.

45. Williamson ME. Major Herbs of Ayurveda. London, UK: Churchill Livingston; 200.

46. Bihaqi SW, Singh AP, Tiwari M. In vivo investigation of the neuroprotective property of Convolvulus pluricaulis in scopolamineinduced cognitive impairments in Wistar rats. Indian J Pharmacol. 2011;43(5):520-525.

47. Sethiya NK, Nahata A, Mishra SH, et al. An update on Shankhpushpi, a cognitive boosting Ayurvedic medicine. Zhong $\mathrm{Xi}$ Yi Jie He Xue Bao. 2009;7(11):1001-1022.

48. Nahata A, Patil UK, Dixit VK. Effect of Convolvulus pluricaulis choisy on learning behavior and memory enhancement activity in rodents. Nat Prod Res. 2008;22(16):1472-1482.

49. Dubey GP, Pathak SR, Gupta BS. Combined effect of Brahmi (Bacopa monniera) and Shankhpushpi (Convolvulus pluricaulis) on cognitive functions. Pharmacopsychoecol. 1994;7:249-251. 